\title{
In Situ Quantification and 3D Reconstruction of Thrombus in the Superficial Femoral Artery Disease Using Optical Coherence Tomography
}

\author{
Mehmet Cilingiroglu ${ }^{1, *}$, Massoud Leesar ${ }^{2}$, Taylor Hoyt ${ }^{3}$, Austin Mcelroy ${ }^{4}$, Thomas Milner ${ }^{4}$, \\ Konstantinos Marmagkiolis ${ }^{5}$, Marc D. Feldman ${ }^{3}$, Vasili Lendel ${ }^{1}$ \\ ${ }^{1}$ Arkansas Heart Hospital, Little Rock, AR, USA/Koc University, School of Medicine, Istanbul, Turkey \\ ${ }^{2}$ University of Alabama at Birmingham, Department of Medicine, Section of Cardiology,Birmingham, AL,USA \\ ${ }^{3}$ University of Texas Health Sciences Center at San Antonio, Department of Medicine, Section of Cardiology, San Antonio, TX, San Antonio, \\ TX, USA \\ ${ }^{4}$ University of Texas at Austin, Cockrell School of Biomedical Engineering, Austin, TX, USA \\ ${ }^{5}$ Citizens Memorial Hospital, Bolivar, MO, USA/University of Missouri, Columbia MO, USA
}

\section{Email address:}

cilingiroglumehmet@gmail.com (M. Cilingiroglu),mleesar@uab.edu (M. Leesar), Taylor.b.hoyt@gmail.com (T. Hoyt), FELDMANM@uthscsa.edu (M. D. Feldman),Mcelroy.austin@gmail.com (A. Mcelroy), terra.laser@gmail.com(T. Milner), c.marmagiolis@gmail.com (K. Marmagkiolis),vlendel@usa.net (V. Lendel)

\section{To cite this article:}

Mehmet Cilingiroglu, Massoud Leesar, Taylor Hoyt, Austin Mcelroy, Thomas Milner, Konstantinos Marmagkiolis, Marc D. Feldman, Vasili Lendel. In Situ Quantification and 3D Reconstruction of Thrombus in the Superficial Femoral Artery Disease Using Optical Coherence Tomography. International Journal of Medical Imaging. Vol. 3, No. 6, 2015, pp. 110-113. doi: 10.11648/j.ijmi.20150306.12

\begin{abstract}
Background: SFA disease accounts for approximately $40 \%$ of the symptomatic peripheral artery disease. Atherosclerosis and thrombosis both contribute in the progression of the peripheral arterial disease. Conventional angiography provides little information about the amount of the thrombus burden. Limited data exists on the use of OCT for the evaluation of PAD. Methods: We performed OCT in three patients with SFA disease who underwent peripheral angiogram from January 2011 - December 2014. Using custom-based imaging software we performed 3D reconstruction and quantification of intraluminal thrombus. Results: We identified thrombus formation in all three patients despite absence of evident thrombosis with invasive angiography. The length of the clot ranged from 12.6 - $47.7 \mathrm{~mm}$ and the volume from 11.19 - $43.26 \mathrm{~mm} 3$ ). Conclusion: Atherosclerosis and thrombosis both contribute in the progression of the peripheral arterial disease. OCT can accurately quantify the length and the volume of the clot burden. Larger studies are needed to assess their clinical importance in the prevention and management of PAD.
\end{abstract}

Keywords: OCT, 3D Reconstruction, PAD

\section{Introduction}

Superficial femoral artery (SFA) disease accounts for approximately $40 \%$ of the symptomatic peripheral artery disease (PAD) and remains a common cause of critical limb ischemia (CLI) and lower extremity amputation. Intra-arterial thrombosis plays a key role in the disease process ${ }^{[1-4]}$. When the disrupted atherosclerotic plaque is exposed to blood, tissue factor and other procoagulant molecules, such as thrombin, an accelerated activation of platelets and formation of fibrin leads to the formation of intraluminal thrombi ${ }^{[5]}$. Conventional angiography provides little information about the amount of the thrombus burden. Intravascular optical coherent tomography (OCT), a novel laser light based imaging modality with a fine axial resolution of 10 micrometer $(\mu \mathrm{m})$ offers superior sensitivity and specificity for the identification and accurate description of intraluminal thrombus ${ }^{[6]}$. However, to date quantification of the total clot burden has not been described.

\section{Methods}

We hypothesized that three-dimension (3D) reconstruction of the OCT studies could yield accurate quantification of the intra-arterial thrombus burden. We retrospectively studied 
three patients who underwent peripheral angiogram and OCT evaluation of the SFA from January 2011 - December 2014. The indication for the peripheral angiogram was "lifestyle-limiting claudication", Rutherford Class III. As there are not currently official guidelines for the use of OCT in the peripheral arterial circulation, OCT was used to optimally visualize and describe the extent of the PAD. The region of interest was the segment of the SFA with obstructive atherosclerosis.

After invasive angiography, OCT imaging of SFA was performed using a St. Jude OCT (Optical coherence tomography) C7-XR system (St. Paul, MN) following power injection of $80 \%$ contrast and $20 \%$ saline at $8 \mathrm{ml} / \mathrm{sec}$ for 5 seconds. Thrombus was defined as a typically jagged protrusion into the luminal space of material with a homogenous texture. In one patient with chronic total occlusion (CTO) of the SFA low pressure balloon angioplasty was performed to re-establish blood flow and allow the passage of the OCT catheter (Figure 1). Using LabVIEW (National Instruments, Austin, TX) custom-based imaging software, each frame of the OCT pullback was analyzed by manually tracing thrombi boundaries (Figure 2). We confirmed that the OCT image quality was optimal and we included only the frames with optimal delineation of the lumen boundaries. All the image pixels of the clot were counted and then converted to a volume by calibrating the catheter to a $1 \mathrm{~mm}$ diameter. Thrombi boundaries were interpolated to provide a $3 \mathrm{D}$ visualization with ImageJ (custom imaging software) and volumetric quantification of the thrombus burden in the region of interest. ImageJ (National Institutes of Health, Bethesda, MD, USA) is a public domain, freely available, cross-platform (e.g., Windows, Mac, Linux) image processing and analysis program developed by the National Institute of Health (NIH) which has been allows three dimension reconstruction and quantification $^{[7,8]}$.
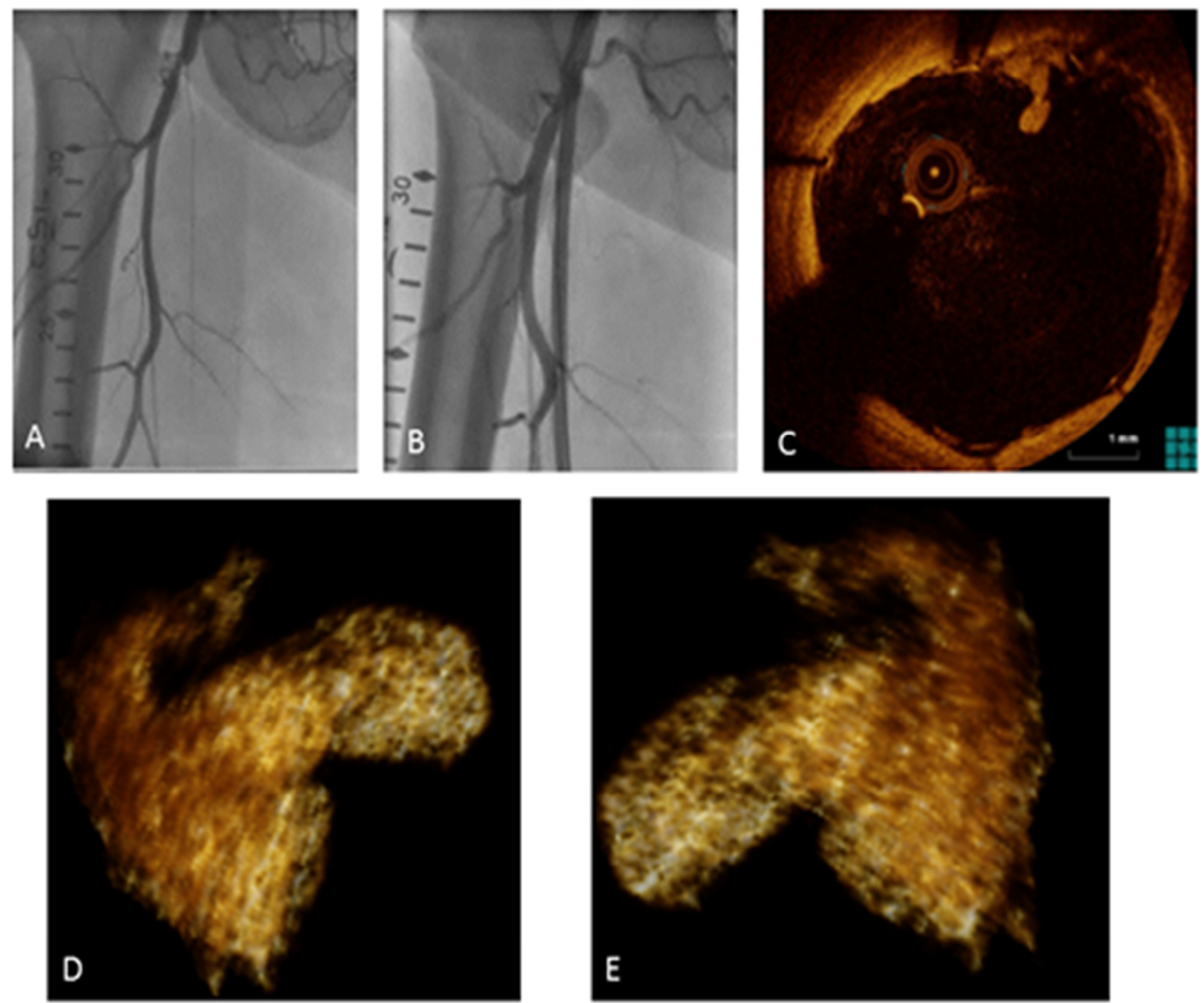

Figure 1. (A-E). Example of a 62 year-old male with a CTO of the SFA. A. Peripheral angiography showing total occlusion of the superficial femoral artery (SFA). B. Successful recanalization of the SFA occlusion following percutaneous intervention with balloon angioplasty and stent placement. C. OCT imaging showing intraluminal clot at 1'o clock position after low-pressure balloon angioplasty. D-E. 3D reconstruction of the clot. CTO: Chronic total occlusion. SFA. Superficial femoral artery. OCT: Optical coherence tomography. 


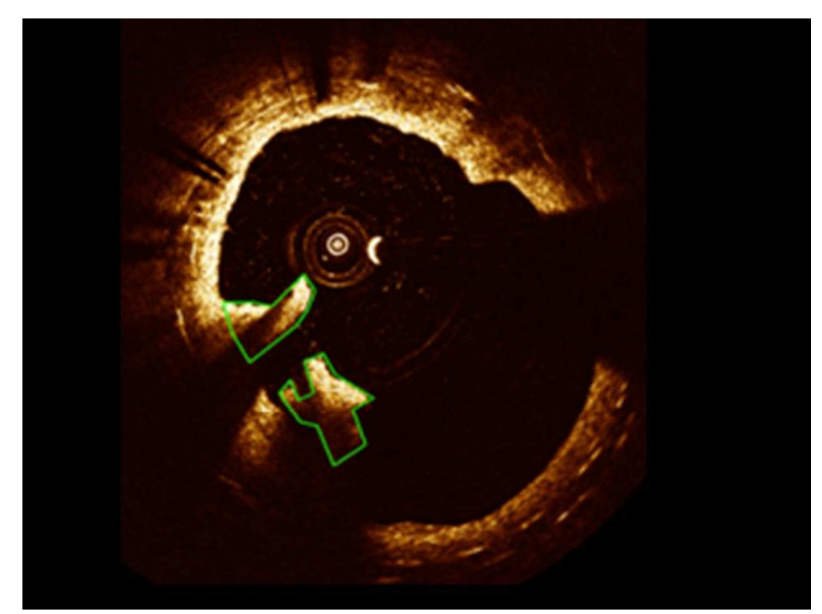

Figure 2. Example of clot quantification using OCT: OCT frame showing thrombi traced in green.

\section{Results}

OCT was successfully performed in all three patients. We identified thrombus formation in all three patients despite absence of evident thrombosis with invasive angiography. After identifying and analyzing the thrombi boundaries via OCT images, we performed a volumetric analysis for quantification and 3D reconstruction of the total clot burden. In the first patient the clot was of a moderate length $(24.4 \mathrm{~mm})$ but large in volume $\left(43.26 \mathrm{~mm}^{3}\right)$. In the second, the clot involved a longer arterial segment $(47.7 \mathrm{~mm})$ but the overall volume was smaller $\left(11.19 \mathrm{~mm}^{3}\right)$. The third patient had a smaller identifiable clot of $12.6 \mathrm{~mm}$ in length and $4.66 \mathrm{~mm}^{3}$ in volume. The results of our analysis (thrombus length, volume and $3 \mathrm{D}$ reconstruction) is shown in table 1 and figure 3.

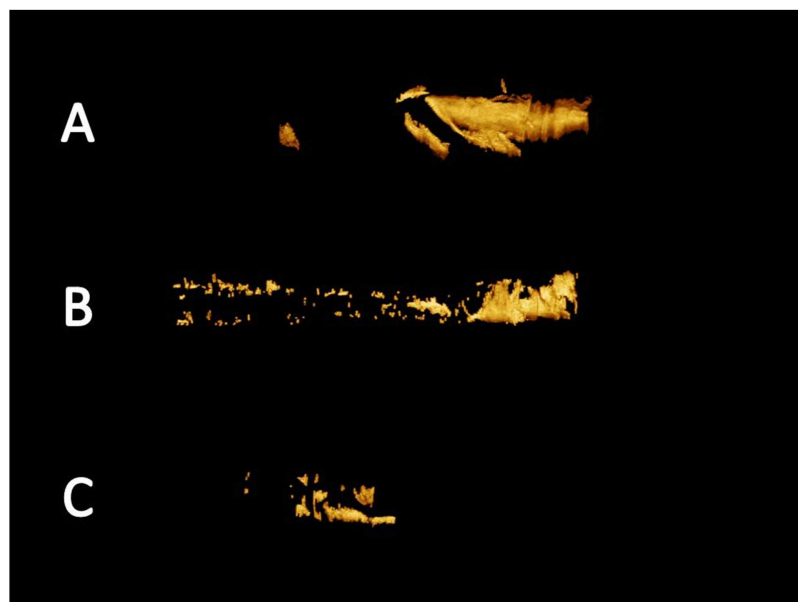

Figure 3. 3D reconstruction of thrombus. A. Large clot B. Moderate clot, $C$. Small clot.

Table 1. Quantification of thrombus length and volume via OCT.

\begin{tabular}{lll}
\hline Patient ID & Length $(\mathbf{m m})$ & Sum volume $\left(\mathbf{m m}^{\mathbf{3}}\right)$ \\
\hline Patient 1 & 24.4 & 43.26 \\
Patient 2 & 47.7 & 11.19 \\
Patient 3 & 12.6 & 4.66 \\
\hline
\end{tabular}

\section{Discussion}

Early autopsy and surgical studies in the 50s and 60 s demonstrated that atherosclerosis and thrombosis coexist in the progression of the "arterial occlusion" $[1,2,9]$. In the TRA2P-TIMI 50 trial, the study of fresh arterial samples obtained at the time of peripheral bypass surgery showed thrombus occlusion which had undergone organization and recanalization $^{[10]}$.

OCT has been extensively used in the coronary circulation for the identification and optimal description of thrombus formation ${ }^{[11]}$. Moreover, the feasibility of OCT in the femoropopliteal arterial circulation has been previously described. In previous studies OCT was used to describe the extent of atherosclerosis, plan consequent intervention and facilitate decision making on the size and length of angioplasty devices (balloons or stents) or to evaluate the results of a previous intervention (restenosis, thrombosis, dissection etc $)^{[6,12,13]}$. To our knowledge, this is the first report of OCT use for the quantification of intraluminal thrombus in the peripheral circulation. We have demonstrated that intravascular OCT can in vivo visualize and quantify thrombus in patients with PAD. The role, type, strength and duration of antiplatelet and anticoagulation after percutaneous interventions is currently unclear ${ }^{[14-18]}$. Information about the total clot burden may influence the type of endovascular strategy, the use of antiplatelet, anticoagulation or thrombolytic agents and the use of embolic protection devices.

\section{Conclusion}

Atherosclerosis and thrombosis both contribute in the progression of the peripheral arterial disease. OCT can accurately quantify the length and the volume of the clot burden. Larger studies are needed to assess their clinical importance in the prevention and management of PAD.

\section{References}

[1] Dunlop GR, Santos R. Adductorcanal thrombosis. The New England journal of medicine. Mar 28 1957; 256(13):577-580.

[2] Lindbom A. Arteriosclerosis and arterial thrombosis in the lower limb; a roentgenological study. Acta radiologica. Supplementum. 1950; 80:1-80.

[3] Tai Z. Transcollateral approach for percutaneous revascularization of complex superficial femoral artery chronic total occlusion. The Journal of invasive cardiology. May 2013; 25(5):E96-100.

[4] Mavor GE. The pattern of occlusion in atheroma of the lower limb arteries; the correlation of clinical and arteriographic findings. The British journal of surgery. Jan 1956; 43(180):352-364.

[5] Jacomella V, Corti N, Husmann M. Novel anticoagulants in the therapy of peripheral arterial and coronary artery disease. Current opinion in pharmacology. Apr 2013; 13(2):294-300. 
[6] Marmagkiolis K, Lendel V, Cawich I, Leesar M, Feldman MD, Cilingiroglu M. Optical coherence tomography to guide below-the-knee endovascular interventions. International journal of cardiology. Oct 20 2014; 176(3):1336-1338.

[7] Gomez-Perez SL, Haus JM, Sheean P, et al. Measuring Abdominal Circumference and Skeletal Muscle From a Single Cross-Sectional Computed Tomography Image: A Step-by-Step Guide for Clinicians Using National Institutes of Health ImageJ. JPEN. Journal of parenteral and enteral nutrition. Sep 212015.

[8] Irving BA, Weltman JY, Brock DW, Davis CK, Gaesser GA, Weltman A. NIH ImageJ and Slice-O-Matic computed tomography imaging software to quantify soft tissue. Obesity. Feb 2007; 15(2):370-376.

[9] Watt JK. Origin of femoro-popliteal occlusions. British medical journal. Dec 18 1965; 2(5476):1455-1459.

[10] Bonaca MP, Scirica BM, Creager MA, et al. Vorapaxar in patients with peripheral artery disease: results from TRA2 \{degrees\}P-TIMI 50. Circulation. Apr 9 2013; 127(14):1522-1529, 1529e1521-1526.

[11] Porto I, Mattesini A, Valente S, Prati F, Crea F, Bolognese L. Optical coherence tomography assessment and quantification of intracoronary thrombus: Status and perspectives. Cardiovascular revascularization medicine: including molecular interventions. Apr-May 2015; 16(3):172-178.

[12] Karnabatidis D, Katsanos K, Paraskevopoulos I, Diamantopoulos A, Spiliopoulos S, Siablis D. Frequency-domain intravascular optical coherence tomography of the femoropopliteal artery. Cardiovascular and interventional radiology. Dec 2011; 34(6):1172-1181.

[13] Negi SI, Rosales O. The role of intravascular optical coherence tomography in peripheral percutaneous interventions. The Journal of invasive cardiology. Mar 2013; 25(3):E51-53.

[14] Efficacy of oral anticoagulants compared with aspirin after infrainguinal bypass surgery (The Dutch Bypass Oral Anticoagulants or Aspirin Study): a randomised trial. Lancet. Jan 29 2000; 355(9201):346-351.

[15] Poredos P, Jezovnik MK. Is aspirin still the drug of choice for management of patients with peripheral arterial disease? VASA. Zeitschrift fur Gefasskrankheiten. Mar 2013; 42(2):88-95.

[16] Johnson WC, Williford WO, Department of Veterans Affairs Cooperative S. Benefits, morbidity, and mortality associated with long-term administration of oral anticoagulant therapy to patients with peripheral arterial bypass procedures: a prospective randomized study. Journal of vascular surgery. Mar 2002; 35(3):413-421.

[17] Ishihara T, Iida O, Awata M, Shiraki T, Nanto S, Uematsu M. Angioscopically apparent large thrombus and uncovered stent struts 6 months after late stent thrombosis of a paclitaxel-coated nitinol drug-eluting stent implanted in the superficial femoral artery. Cardiovascular intervention and therapeutics. Jan 2014; 29(1):82-85.

[18] Hanna EB. Dual antiplatelet therapy in peripheral arterial disease and after peripheral percutaneous revascularization. The Journal of invasive cardiology. Dec 2012; 24(12):679-684. 